\title{
Ipsilateral Supracondylar Fracture and Forearm Bone Injury in Children: A Retrospective Review of Thirty one Cases
}

\author{
Dhoju D, Shrestha D, Parajuli N, Dhakal G, Shrestha R
}

Department of Orthopaedics and traumatology

Dhulikhel Hospital-Kathmandu University Hospital

Dhulikhel, Nepal

Corresponding Author

Dr Dipak Shrestha

Department of Orthopaedics and traumatology

Dhulikhel Hospital-Kathmandu University Hospital

Dhulikhel, Nepal.

E-mail: dsmsortho@gmail.com

Mobile No: 9851073353

Citation

Dhoju D, ShresthaD, Parajuli N, Dhakal G, Shrestha R. Ipsilateral supracondylar fracture and forearm bone injury in children: a retrospective review of thirty one Cases. Kathmandu Univ Med J 2011;34(2)11-6.

\begin{abstract}
Background

Supracondylar fracture and forearm bone fracture in isolation is common musculoskeletal injury in pediatric age group But combined supracondylar fracture with ipsilateral forearm bone fracture, also known as floating elbow is not common injury. The incidence of this association varies between $3 \%$ and $13 \%$. Since the injury is rare and only limited literatures are available, choosing best management options for floating elbow is challenging.
\end{abstract}

\section{Method}

In retrospective review of 759 consecutive supracondylar fracture managed in between July 2005 to June 2011, children with combined supracondylar fracture with forearm bone injuries were identified and their demographic profiles, mode of injury, fracture types, treatment procedures, outcome and complications were analyzed.

Result

Thirty one patients (mean age 8.91 yrs, range 2-14 yrs; male 26; left side 18) had combined supracondylar fracture and ipsilateral forearm bone injury including four open fractures. There were 20 (64.51\%) Gartland type III (13 type IIIA and 7 type III B), seven (22.58 \%) type II, three (9.67 \%) type I and one (3.22 \%) flexion type supracondylar fracture. Nine patients had distal radius fracture, six had distal third both bone fracture, three had distal ulna fracture, two had mid shaft both bone injury and one with segmental ulna with distal radius fracture. There were Monteggia fracture dislocation, proximal ulna fracture, olecranon process fracture, undisplaced radial head fracture of one each and two undisplaced coronoid process fracture. Type I supracondylar fracture with undisplaced forearm were treated with closed reduction and long arm back slab or long arm cast. Displaced forearm fracture required closed reduction and fixation with Kirschner wires or intramedullary nailing. Nineteen patients with Gartland type III fracture underwent operative intervention. Among them nine had closed reduction and $\mathrm{K}$ wire fixation for both supracondylar fracture and forearm bone injury. One patient with closed reduction and long arm cast application for both type III supracondylar fracture and distal third radius fracture developed impending compartment syndrome and required splitting of cast, remanipulation and Kirschner wire fixation. There were three radial nerve, one ulnar nerve and one median nerve injury and two postoperative ulnar nerve palsy. Three patients had pin tract related complications. Among type III, 16 (80\%) patients had good to excellent, two had fair and one gad poor result in terms of Flynn's criteria in three months follow up

\section{Conclusion}

Displaced supracondylar fracture with ipsilateral displaced forearm bone injuries need early operative management in the form of closed reduction and percutaneous pinning which provides not only stable fixation but also allows close observation for early sign and symptom of development of any compartment syndrome.

\section{KEYWORDS}

Floating elbow; Forearm bone injury; Ipsilateral fracture; Supracondylar fracture 


\section{INTRODUCTION}

Supracondylar fracture and forearm bone fracture in isolation is common musculoskeletal injury in pediatric age group. Supracondylar fracture consists of $50 \%$ to $70 \%$ of elbow injury; fracture of shaft radius or ulna or both accounts for 5 to $10 \%$ all pediatric fracture and distal forearm bone fractures comprise $75 \%$ of all forearm fracture in children. ${ }^{1}$ But combined supracondylar fracture with ipsilateral forearm bone fracture, also known as floating elbow is not common injury. ${ }^{2}$ Some of the largest reported series of supracondyalr fracture and even some textbooks of pediatric musculoskeletal injury does not mention about such a combination of injury. ${ }^{3}$ The incidence of this association varies between $3 \%$ and $13 \%{ }^{4}$

Various combinations of treatment options has been suggested for this injury such as primary closed reduction of both fracture and long arm cast application or olecranon pin traction till swelling subsides and delayed long arm cast application or pinning supracondylar fracture and short arm cast application for forearm bone injury or closed reduction and percutaneous pinning for both injuries. ${ }^{5-8}$ Because of rarity of injury and limited availability of literature, choosing best management options for floating elbow is challenging. We therefore retrospectively reviewed treatment and outcome of ipsilateral supracondyalar fracture and forearm bone fracture treated over six years period in our institution.

\section{METHOD}

All pediatric supracondylar fractures, treated in Kathmandu University Hospital, Dhulikhel Hospital in between July 2005 to June 2011 were retrospectively reviewed from medical case records. Among them, supracondylar fracture with ipsilateral forearm bone injuries were identified and evaluated for demographic data, mechanism of injury, treatment method, associated complications and outcomes. Final outcomes were assessed according to Flynn's criteria.

Patients who presented with supracondylar fracture Gartland type I with undisplaced forearm bone injuries were managed in emergency room with or without mild sedation with posterior long arm back slab alone or along with short arm cast. Patients (displaced supracondylar fracture or displaced forearm bone fracture) requiring manipulation or Kirschner $(\mathrm{K})$ wire fixation were posted for next available surgical day after temporary immobilization of the limb and pain control medications. Open fractures were managed on the same day of presentation with wound debridemet, fracture fixation and intravenous antibiotics.

Forearm bone fracture were reduced and fixed first before supracondylar fracture. In patients with both bone metaphyseal fracture, only radius was fixed with crossed $\mathrm{K}$ wires. First $\mathrm{K}$ wire was passed through or just proximal to radial styloid process to avoid growth plate and advanced proximally medially to cross the fracture site. Another $\mathrm{K}$ wire was passed from Lister's tubercle to volar and lateral side. Supracondylar fracture were reduced with standard technique and fixed with two crossed $\mathrm{K}$ wires or two lateral $\mathrm{K}$ wires with or without supplementation of third $\mathrm{K}$ wire depending upon stability and communiation of fracture site. Utmost care was taken not to injure ulnar nerve during passing medial wire by rolling over nerve posteriorly or making a small stab incision and passing a haemostat to palpate medial epicondyle. All the $\mathrm{K}$ wires bent outside skin
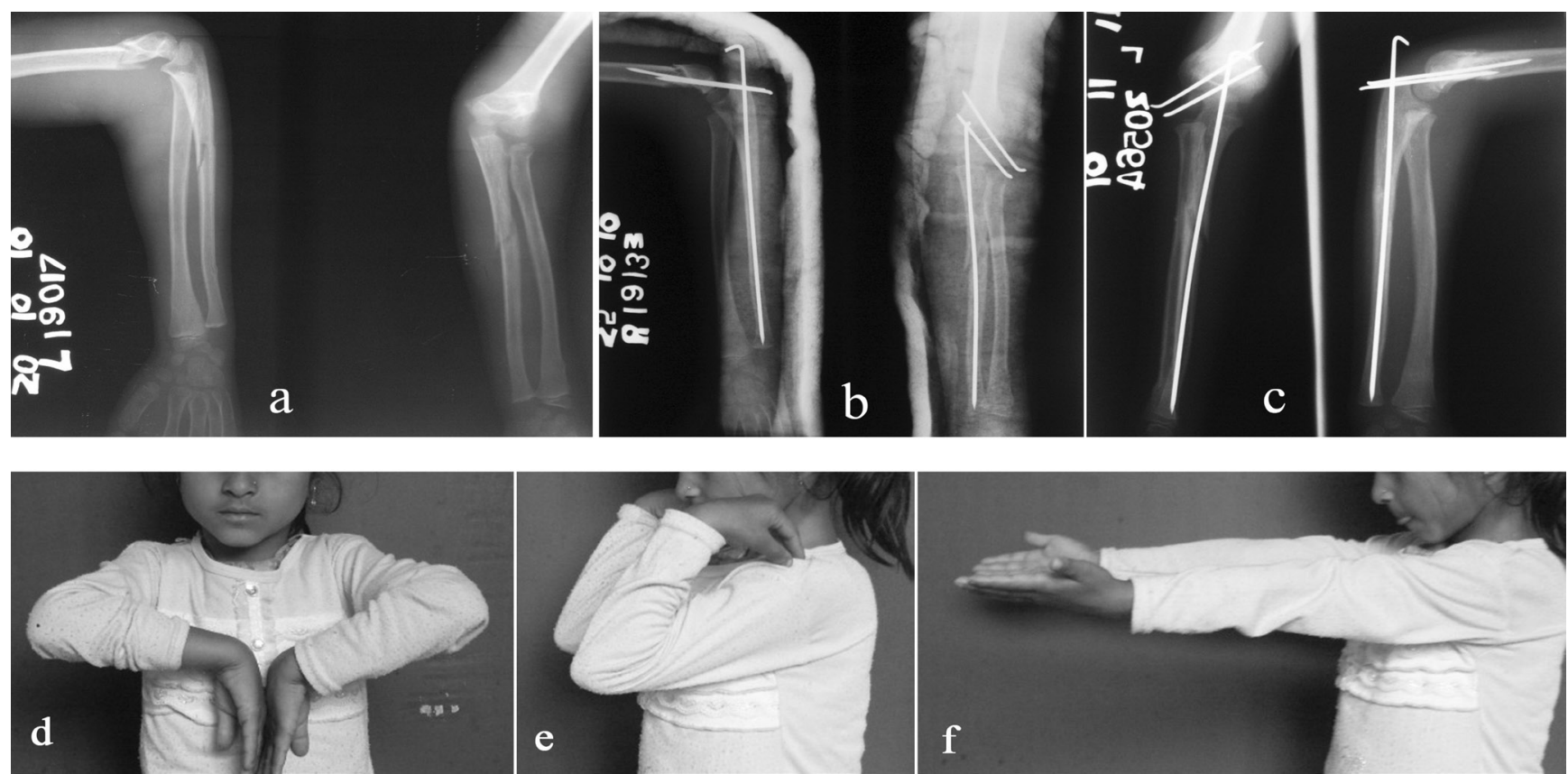

Figure 1. Closed reduction and per cutaneous $\mathrm{K}$ wire fixation for supracondylar fracture Gartland type II and closed reduction and intramedullary nailing for ipsilateral ulna shaft fracture (note: Torrus fracture of distal radius) (a) pre operative (b) post operative (c) follow up at 6 weeks (d, e, f) final excellent clinical outcome 
Table 1. Demographic profiles of patients and details of injury, procedure and complications.

\begin{tabular}{|c|c|c|c|c|c|c|c|c|c|}
\hline Age & M & $\begin{array}{l}\text { Mechanism of } \\
\text { injury } \\
\text { Fall }\end{array}$ & $\mathrm{L}$ & $\begin{array}{l}\text { Clinical } \\
\text { type } \\
\text { Closed }\end{array}$ & $\begin{array}{l}\text { Gartland } \\
\text { type } \\
\text { Flexion }\end{array}$ & $\begin{array}{l}\text { Ipsilateral forearm } \\
\text { injury } \\
\text { Monteggia fracture } \\
\text { dislocation }\end{array}$ & Nerve injury & $\begin{array}{l}\text { Procedure } \\
\text { ORPP }\end{array}$ & Remarks \\
\hline 8 & M & Fall & L & Closed & I & Both bone midshaft & & CRLAC & \\
\hline 10 & M & Fall & L & Closed & I & Distal radius SH type II & & LABS and CRPP & \\
\hline 6 & $M$ & Fall & $R$ & Closed & I & Undisplaced olecranon & & LABS & \\
\hline 12 & M & Playground injury & $\mathrm{L}$ & Closed & ॥ & Distal 3rd both bone & & LABS & \\
\hline 10 & M & Playground injury & L & Closed & II & Distal 3rd both bone & & LABS & \\
\hline 8 & M & Fall & $\mathrm{R}$ & Closed & II & Distal 3rd radius & & LABS & $\begin{array}{l}\text { Remanipulation and } \\
\text { cast application for } \\
\text { distal radius }\end{array}$ \\
\hline 8 & $\mathrm{~F}$ & Fall & L & Closed & II & $\begin{array}{l}\text { Distal radius and seg- } \\
\text { mental ulna }\end{array}$ & & $\begin{array}{l}\text { CRPP, ulna nail- } \\
\text { ing and LABS }\end{array}$ & \\
\hline 3 & $\mathrm{~F}$ & Fall & $\mathrm{R}$ & Closed & II & Undisplaced coronoid & & LABS & \\
\hline 3 & $\mathrm{~F}$ & Fall & $\mathrm{R}$ & Closed & ॥ & Undisplaced coronoid & & LABS & \\
\hline 7 & M & Playground injury & $\mathrm{R}$ & Closed & ॥ & Undisplaced radial head & & LABS & \\
\hline 12 & M & Fall & L & Closed & III A & Distal 3 rd radius & & CRPP & \\
\hline 11 & M & Playground injury & L & Closed & III A & Distal radius SH type I & & CRPP & \\
\hline 11 & M & Fall & L & Closed & III A & Distal radius torrus & & ORPP and LABS & \\
\hline 2 & M & Fall & $\mathrm{L}$ & Closed & IIIA & Distal 3rd both bone & & CRPP & \\
\hline 8 & M & Fall & L & Closed & IIIA & Distal 3rd both bone & & $\begin{array}{l}\text { CRPP and Short } \\
\text { arm cast }\end{array}$ & $\begin{array}{l}20 \text { degree angulation } \\
\text { of radius }\end{array}$ \\
\hline 4 & $\mathrm{~F}$ & Fall & $\mathrm{R}$ & Closed & IIIA & Distal 3rd radius & & CRPP & $\begin{array}{l}\text { Post op. ulnar nerve } \\
\text { palsy, Discharge from } \\
\text { pin tract }\end{array}$ \\
\hline 9 & M & Fall & $\mathrm{R}$ & Closed & IIIA & & & $\begin{array}{l}\text { CRPP and Short } \\
\text { arm cast }\end{array}$ & \\
\hline 16 & M & Fall & L & Open & IIIA & Distal 3rd radius & & ORPP & \\
\hline 9 & M & Fall & $R$ & Closed & IIIA & Distal 3rd radius & & CRLAC & \\
\hline 10 & M & Fall & $\mathrm{R}$ & Closed & IIIA & $\begin{array}{l}\text { Distal radius SH type III, } \\
\text { distal ulna }\end{array}$ & Radial & CRPP & $\begin{array}{l}\text { Fingers swelling, } \\
\text { plasters splited and } \\
\text { remanipulation and } \mathrm{K} \\
\text { wire fixation }\end{array}$ \\
\hline 10 & M & Fall & $\mathrm{R}$ & Open & IIIA & Distal radius SH type I & Radial & ORPP & \\
\hline 7 & M & Fall & L & Closed & IIIA & Distal 3rd ulna & & ORPP & \\
\hline 4 & $\mathrm{~F}$ & Fall & L & Closed & IIIA & Proximal ulna & & CRPP & \\
\hline 10 & M & Fall & L & Closed & IIIB & Distal 3rd both bone & & CRPP & Loosening of $\mathrm{K}$ wire \\
\hline 8 & M & Fall & $\mathrm{R}$ & Open & IIIB & Distal 3rd both bone & Radial & ORPP & Jones transfer \\
\hline 3 & $\mathrm{~F}$ & Fall & $\mathrm{R}$ & Open & IIIB & Both bone midshaft & Ulnar & ORPP & Ulnar nerve repair \\
\hline 2 & M & Fall & L & Closed & IIIB & Distal 3rd radius & & CRPP & \\
\hline 11 & M & Fall & L & Closed & IIIB & Distal 3rd ulna & & ORPP & Loosening of $\mathrm{K}$ wire \\
\hline 11 & M & Fall & L & Closed & IIIB & Distal 3rd ulna & & & $\begin{array}{l}\text { Post op. ulnar nerve } \\
\text { palsy }\end{array}$ \\
\hline 10 & M & Fall & L & Closed & IIIB & Distal 3rd radius & & CRPP & \\
\hline
\end{tabular}

(SH: Salter and Harris; CRPP: closed reduction and per cutaneous K wire fixation; ORPP: open reduction and per cutaneous $\mathrm{K}$ wire fixation; LABS: long arm back slab; CRLAC: closed reduction and long arm cast)

for easy removal on OPD basis during follow up. Limb was splinted with posterior long arm back slab for three to six weeks. Follow up visits were made to evaluate progress of fracture healing, fracture displacement or any signs of infection.

\section{RESULT}

In a series of 749 consecutive pediatric supracondylar fractures, 31(4.13\%) children (mean age 8.91 yrs, range 2-14 yrs) had supracondylar fracture with ipsilateral forearm 
bone injury. Twenty five children were male and six were female. Left side was involved in 18 patients and right side in 13 patients. There were two open supracondylar injuries of Gustillo grade I; one of Gustillo grade II and one both bone distal third forearm fracture of Gustillo grade II.

There were 20 (64.51\%) Gartland type III (13 type IIIA and seven type III B), seven (22.58 \%) type II, three (9.67\%) type I and one (3.22\%) flexion type supracondylar fracture. Among forearm bone injury, nine were distal radius fracture, six were distal third both bone fracture, three were distal ulna fracture, two were midshaft both bone injury and one with segmental ulna with distal radius fracture. There were Monteggia fracture dislocation, proximal ulna fracture, olecranon process fracture, undisplaced radial head fracture of one each and two undisplaced coronoid process fracture. Distal growth plate injuries were found in four patients (Salter Harris type I: 2 distal radius, Salter Harris type II: 1 distal radius and Salter Harris type III: 1 distal radius associated with distal third ulna fracture).

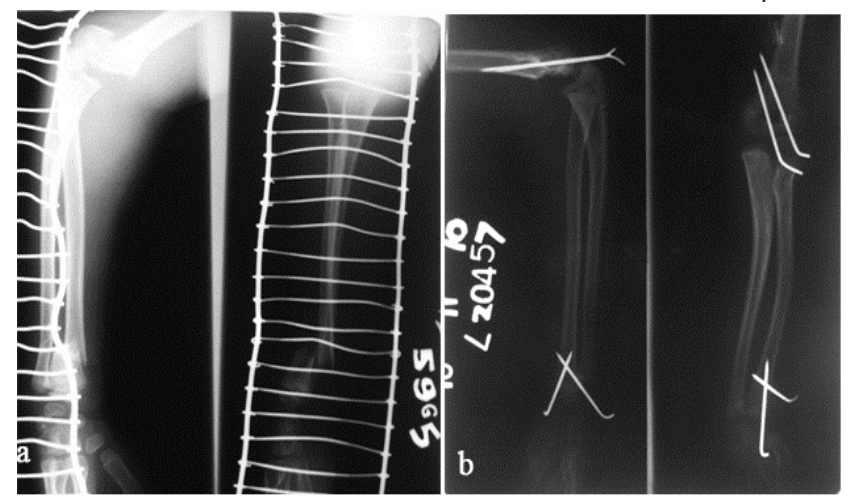

Figure 2. Closed reduction and per cutaneous $\mathrm{K}$ wire fixation for supracondylar fracture Gartland type III with ipsilateral distal metaphyseal both bone forearm fracture (a) pre reduction (b) follow up at 3 weeks

Two patients with Gartland type I supracondylar fracture with undisplaced olecranon fracture and both bone midshaft forearm fracture were treated with closed reduction followed by long arm back slab and longarm cast respectively. Another patient with type I supracondylar

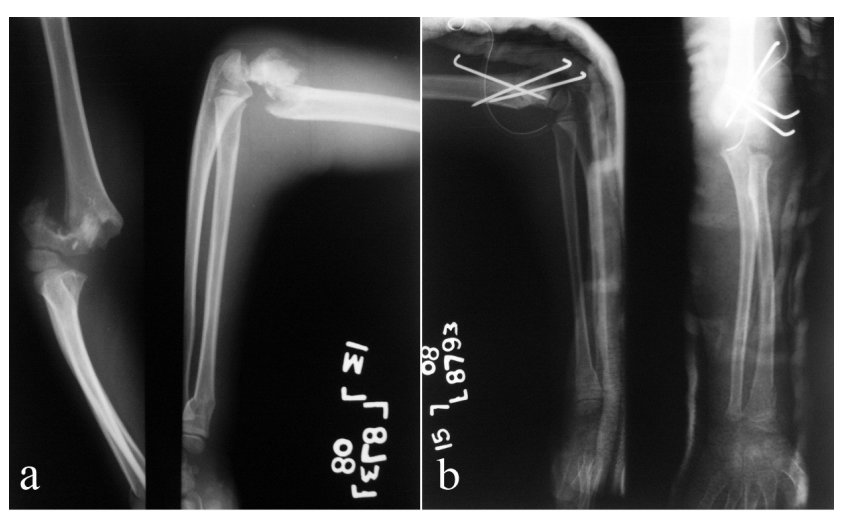

Figure 3. Open reduction and per cutaneous $\mathrm{K}$ wire fixation for supracondylar fracture Gartland type III and closed reduction and long arm slab for ipsilateral undisplaced distal metaphyseal radius fracture (a) pre operative (b) post operative fracture had ipsilateral Salter Harris type II distal radius fracture which was managed with closed reduction and per cutaneous $\mathrm{K}$ wire pinning and long arm posterior slab.

Among seven Gartland type II supracondylar fracture, one had ipsilateral distal radius fracture which required closed reduction and $\mathrm{K}$ wire fixation and segmental ulna fracture which was managed with closed reduction and intramedullary nailing. (Fig.1) Rest of type II fracture which was associated with undisplaced coronoid fracture or radial head fracture or undisplaced distal both bone fracture were managed with closed reduction and long arm back slab.

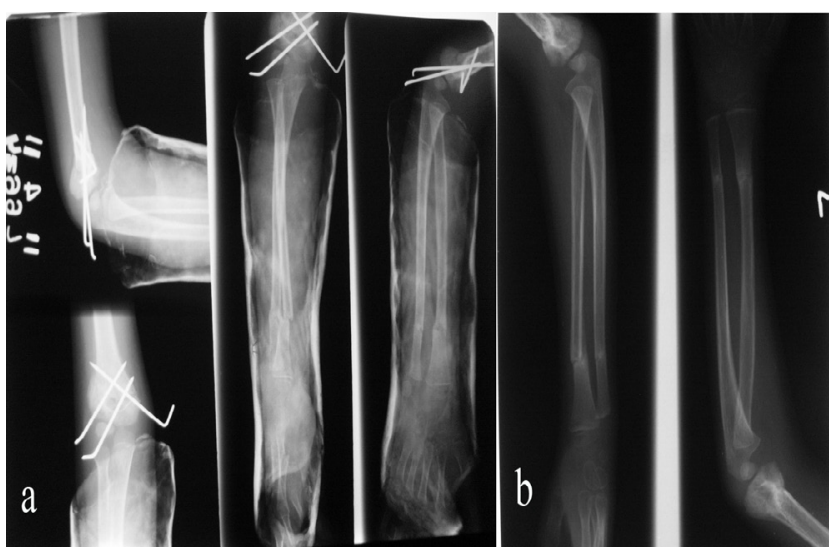

Figure 4. Closed reduction and per cutaneous $\mathrm{K}$ wire fixation for supracondylar fracture Gartland type III and closed reduction and short arm cast for ipsilateral distal forearm both bone fracture (a) post operative (b) follow up at 6 weeks showing radial malunion

Nineteen patients with Gartland type III fracture underwent operative intervention. Among them nine had closed reduction and $\mathrm{K}$ wire fixation for both supracondylar fracture and forearm bone injury (Fig. 2) which included two patients with growth plate injury of distal radius. Two patients had closed reduction and $\mathrm{K}$ wire fixation for supracondylar fracture and closed reduction and short arm cast for forearm bone injuries. Open procedure were required in eight children with supracondylar fracture (Fig. 3) including two open fractures. Only one patient with Gartland type III was managed with closed reduction and long arm cast application for both supracondylar fracture and distal third radius fracture but developed impending compartment syndrome which required splitting of cast, remanipulation and $\mathrm{K}$ wire fixation once swelling subsided. Patient with flexion type of supracondylar fracture was associated with Monteggia fracture dislocation which was managed with open reduction for supracondylar fracture and closed intramedullary nailing for ulna fracture followed by long arm slab. There were five (16.12\%) patients with nerve injuries (Radial nerve: 3 , Ulnar nerve: 1 and Median nerve: 1 ) among them three were associated with open supracondylar fracture among which one under went ulnar nerve repair. One patient with radial nerve injury underwent tendon transfer procedure as there was no sign of recovery even after 6 months follow up. Two patients 
developed postoperative ulnar nerve palsy which recovered in subsequent follow up. No vascular injury was identified in the present study. Three patients had pin tract related complications which included discharge in one patient and loosening of distal radius pin in two patients.

All Gartland type I and II supracondylar fracture with ipsilateral forearm bone injury had excellent outcome and among type III, 16 (80\%) patients had good to excellent result in terms of Flynn's criteria in three months follow up. Fair result was seen in patient in two and poor result in one patient

\section{DISCUSSION}

Supracondylar fracture in children is usually due to fall on outstretched with elbow hyperextended but when additional force is transmitted through to wrist, it is associated with forearm bone injury. Greater amount of force is required for multiple bony injury of the ipsilateral limb. When forearms fracture is proximal to the junction of the middle and distal third, the lever arm of the proximal forearm segment is considered too short to generate the moment of force required to produce ipsilateral humeral fracture. ${ }^{9}$ But this mechanism does not explain other forearm bone injuries around the elbow such as coronoid process, olecranon process or Monteggia fracture dislocation .associated with ipsilateral supracondylar fracture. The alternative mechanism of injury has been proposed by Taylor AK et al which mention that fall on outstretched hand with partially flexed elbow where normal anteversion of the distal humerus converts compressive force into shearing force which can explain other elbow injuries including distal and midshaft forearm bone fractures. ${ }^{10}$ In the present series, there were two cases of coronoid process fracture, one radial head fracture, one olecranon process fracture and one Monteggia fracture dislocation and one proximal ulna fracture with ipsilateral supracondylar fracture.

Combined supracondylar fracture with forearm bone injury is considered high energy fracture. The force is so much that single fracture could not dissipate all the energy of trauma and there are increased chance of soft tissue injury including nerves and vessels. ${ }^{11}$ Though we did not encounter any vascular injury, there were $16.12 \%$ (5 cases) of nerve injury which is more than reported incidence of nerve injury $(7.7 \%)$ with isolated supracondylar fracture. Similarly incidence of open fracture (12.9\%) in the present study is also higher than usual incidence of $1 \%$ in supracondylar fracture in isolation. ${ }^{2}$

Management option for displaced supracondylar fracture has evolved from conservative management with closed reduction and posterior back slab, olecranon traction to closed reduction and percutaneous $\mathrm{K}$ wire fixation. Various techniques of $\mathrm{K}$ wire fixation such as crossed $\mathrm{K}$ wires, lateral two $\mathrm{K}$ wires have been described in literature for better biomechanical stability and to decrease chance of potential iatrogenic nerve injury. ${ }^{12}$ Similarly, distal both bone forearm fracture which is mostly treated with closed reduction and cast application has recently change in trends towards primarily closed reduction and percutaneous $\mathrm{K}$ wire fixation to prevent redisplacement of the fracture in the cast. ${ }^{13}$ The incidence of redisplacement is reported up to $7 \%$ to $25 \%$ with various studies. ${ }^{14,15}$ But combined supracondylar fracture and forearm bone fracture which is described as difficult to treat in literature as contrast to fracture in isolation, are associated with high energy trauma, increased incidence of potential neurovascular damage and compartment syndrome. Biyani A et al reported primarily posterior slab for supracondylar facture and short arm cast for forearm fracture and olecranon pin traction or $\mathrm{K}$ wire fixation for supracondylar fracture only when closed reduction failed. ${ }^{5}$ Similarly Reed et al also had also used plaster slab for both injury after fracture reduction. Both of these study reported cubitus varus deformity with incidence of $20 \%$ but did not find any compartment syndrome. ${ }^{5,16}$ Stanitski $\mathrm{CL}$ et al advocated use of olecranon pin traction or closed pinning for supracondylar fracture and short arm cast for forearm fracture. ${ }^{6}$ Similarly, Williamson DM et al managed the supracondylar fracture by traction or manipulative reduction and percutaneous pinning and the forearm fractures were managed by reduction and casting but is associated with prolonged hospitalization and hence increased financial and psychological burden. ${ }^{17}$ But, because of higher incidence of compartment syndrome associated with floating elbow which is reported as high as up to $33 \%$, various authors have recommended pinning for both fracture which allows close observation of neurovascular structure and also reduces chances of redisplacement of fracture in cast or slab. ${ }^{4,11,18,19}$ Ring $D$ et al found two patients with compartment syndrome and four patients with incipient compartment syndrome in ten patients treated with closed reduction and cast immobilization where as none of the patients with percutaneous pinning of the fractures developed compartment syndrome. ${ }^{11}$ Roposch A et al reported redisplacement in three cases among 18 patients with forearm fractures treated with cast while none in 29 cases managed with percutaneous pinning. ${ }^{4}$ The present series found one case of impending compartment syndrome after conservative management with cast requiring splitting of cast, remanipulation and pinning later on and similarly one case of malunited radius fracture with 20 degree anterior angulation in patient with short arm cast for distal third both bone fracture. (Fig. 4)

Priority of reduction of stabilization of supracondylar fracture or forearm injury first varies among authors though no definitive study has been done in terms of outcome and complications. Those who advocate supracondylar fracture first assume that maintenance of reduction and access to the limb for neurovascular monitoring, dressings and the closure of open fractures may be difficult if the forearm fracture is treated first. ${ }^{5,9}$ But in the present study, we 
reduced and fixed forearm bone fracture first because we believe that leaving dangling forearm during reduction of supracondylar fracture can cause more soft tissue injury of forearm and can increase chance of compartment syndrome. Similarly pronation or supination of forearm required to maintain reduction of supracondylar fracture will be safe and easy if we reduce and fix forearm bone injury first.

Final outcome in the present study as assessed by Flynn's criteria ( $80 \%$ good or excellent among Gartland type III supracondylar fracture) is comparable to Harrington P et al (83\% good to or excellent). ${ }^{3}$

\section{REFERENCES}

1. Herring JA, editor. Upper extremity injuries. In: Tachdjian's Pediatric Orthopaedics. 4th ed. Vol. 3. Philadelphia: W.B. Saunders; 2008.

2. Skaggs DL, Flynn JF: Supracondylar Fracture of the Distal Humerus. In: Beaty JH, Kasser JR, (editors) Rockwood and Wilkins Fractures in Children, 7th Edition Vol. 3. Philadelphia, Lippincott William and Wilkins; 2010. 487-531.

3. Harrington $P$, Sharif I, Fogarty EE, Dowling FE, Moore DP. Management of the floating elbow injury in children. Simultaneous ipsilateral fractures of the elbow and forearm. Arch Orthop Trauma Surg.2000;120:205-8.

4. Roposch A, Reis M, Molina M, Davids J, Stanley E, Wilkins K, et al. Supracondylar fractures of the humerus associated with ipsilateral forearm fractures in children: A report of forty seven cases. J Pediatr Orthop. 2001;21:307-12.

5. Biyani A, Gupta SP, Sharma JC. Ipsilateral supracondylar fracture of the humerus and forearm bones in children. Injury. 1989;20:203-7.

6. Stanitski CL, Micheli LJ. Simultaneous ipsilateral fractures of the arm and forearm in children. Clin Orthop Relat Res. 1980;153:218-22.

7. Tabak AY, Celibi L, Muratli HH, Yagmurlu MF, Aktekin CN, Biçimoglu A. Closed reduction and percutaneous fixation of supracondylar fracture of the humerus and ipsilateral fracture of the forearm in children. J Bone Joint Surg Br. 2003;85:1169-72

8. Suresh SS. Management of "floating elbow" in children. Indian J Orthop. 2007; 41(4): 386-389.

9. Templeton PA, Graham HK. The 'Floating elbow' in childrenSimultaneous supracondylar fractures of the humerus and of the forearm in the upper limb. J Bone Joint Surg Br. 1995;77:791-6.

\section{CONCLUSION}

Ipsilateral supracondylar fracture and forearm bone injuries are result of high energy trauma and associated with higher incidence of nerve injury. Displaced supracondylar fracture with ipsilateral displaced forearm bone injuries need early operative management in the form of closed reduction and percutaneous pinning which provides not only stable fixation but also allows close observation for early sign and symptom of development of any compartment syndrome.

10. Taylor KA, Junewick JJ. Simultaneous ipsilateral elbow and forearm fractures in children: a retrospective review. Emergency Radiology. 2002;9: 314-316.

11. Ring D, Waters PM, Hotchkiss RN, Kasser JR. Pediatric floating elbow. J Pediatr Orthop.2001;21:456-59.

12. Pandey S, Shrestha D, Gorg M, Singh GK, Singh MP. Treatment of supracondylar fracture of the humerus (type IIB and III) in children: A prospective randomized controlled trial comparing two methods. Kathmandu University Medical Journal.2008;6(23): 310-318.

13. Sherestah D, Dhoju D, Parajuli $N$ et al. Management of pediatric displaced distal metaphyseal fracture: Comparison between cast immobilization and percutaneous Kirschner wire fixation. Nepal Orthopedic Association Journal (accepted).

14. Proctor MT, Moore DJ, Paterson JM. Redisplacement after manipulation of distal radial fractures in children. J Bone Joint Surg 1993;75B:453-454.

15. Voto SJ, Weiner DS, Leighley B. Redisplacement after closed reduction of forearm fractures in children. J Pediatr Orthop 1990;10:79-84.

16. Reed FE, Jr, Apple DF. Ipsilateral fractures of the elbow and forearm. South Med J.1976;69:149-51.

17. Williamson DM, Cole WG. Treatment of ipsilateral supracondylar and forearm fractures in children. Injury. 1992;23:159-61.

18. Hwang RW, de Witte PB, Ring D. Compartment syndrome sssociated with distal radial fracture and ipsilateral elbow injury. J Bone Joint Surg Am. 2009;91:642-5.

19. Blakemore LC, Cooperman DR, Thompson GH, Wathey C, Ballock RT. Compartment syndrome in ipsilateral humerus and forearm fractures in children. Clin Orthop Relat Res. 2000;376:32-8. 